\title{
Metastatic Infiltrating Bladder Urothelial Carcinoma, Micropapillary Variant
}

National Cancer Institute

\section{Source}

National Cancer Institute. Metastatic Infiltrating Bladder Urothelial Carcinoma,

Micropapillary Variant. NCI Thesaurus. Code C157767.

Bladder micropapillary variant urothelial carcinoma that has spread to another anatomical site. 